\title{
A Fast Parallel Elliptic Curve Multiplication Resistant against Side Channel Attacks
}

\author{
Tetsuya Izu ${ }^{1, \star}$ and Tsuyoshi Takagi ${ }^{2}$ \\ 1 Fujitsu Laboratories Ltd., \\ 4-1-1, Kamikodanaka, Nakahara-ku, Kawasaki, 211-8588, Japan \\ izu@flab.fujitsu.co.jp \\ 2 Technische Universität Darmstadt, Fachbereich Informatik, \\ Alexanderstr.10, D-64283 Darmstadt, Germany \\ ttakagi@cdc.informatik.tu-darmstadt.de
}

\begin{abstract}
This paper proposes a fast elliptic curve multiplication algorithm applicable for any types of curves over finite fields $\mathbb{F}_{p}$ ( $p$ a prime), based on [Mon87], together with criteria which make our algorithm resistant against the side channel attacks (SCA). The algorithm improves both on an addition chain and an addition formula in the scalar multiplication. Our addition chain requires no table look-up (or a very small number of pre-computed points) and a prominent property is that it can be implemented in parallel. The computing time for $n$-bit scalar multiplication is one ECDBL $+(n-1)$ ECADDs in the parallel case and $(n-1)$ ECDBLs $+(n-1)$ ECADDs in the single case. We also propose faster addition formulas which only use the $x$-coordinates of the points. By combination of our addition chain and addition formulas, we establish a faster scalar multiplication resistant against the SCA in both single and parallel computation. The improvement of our scalar multiplications over the previous method is about $37 \%$ for two processors and $5.7 \%$ for a single processor. Our scalar multiplication is suitable for the implementation on smart cards.
\end{abstract}

\section{Introduction}

In recent years, several elliptic-curve based cryptosystems (ECC) have been included in many standards [ANSIIEEE NIST SECIWAP]. The key length of ECC is currently chosen smaller than those of the RSA and the ElGamal-type cryptosystems. The small key size of ECC is suitable for implementing on low-power mobile devices like smart cards, mobile phones and PDAs (Personal Digital Assistants, such as Palm and Pocket PC). Let $E(K)$ be an elliptic curve over a finite field $K=\mathbb{F}_{p}$ ( $p$ a prime). The dominant computation of the encryption/decryption and the signature generation/verification of ECC is the scalar

\footnotetext{
* This work was done while the first author was staying at the Centre for Appllied Cryptographic Research (CACR), University of Waterloo and part of this work was done while the first author was visiting Fachbereich Informatik, Technische Universität Darmstadt.
}

D. Naccache and P. Paillier (Eds.): PKC 2002, LNCS 2274, pp. 280-296 2002

(C) Springer-Verlag Berlin Heidelberg 2002 
multiplication $d * P$, where $P \in E(K)$ and $d$ is an integer. It is usually computed by combining an adding $P+Q$ (ECADD) and a doubling $2 * P$ (ECDBL), where $P, Q \in E(K)$. Several algorithms have been proposed to enhance the running time of the scalar multiplication Gor98 CMO98. The choice of the coordinate system and the addition chain is the most important factor. A standard way in [IEEE] is to use the Jacobian coordinate system and the addition-subtraction chain. Some efficient addition chains use a table look-up method. It is useful for software implementation but not for smart cards because the cost of the memory spaces is expensive and an I/O interface to read the table is relatively slow.

This paper proposes a fast multiplication which is applicable for any type of elliptic curves over finite fields $K=\mathbb{F}_{p}$ ( $p$ a prime). The algorithm improves both the addition chain and the addition formula in the scalar multiplication. Our addition chain requires no table look-up (or a very small table) and a prominent property of our addition chain is that it can be implemented in parallel 1 The latency of the scalar multiplication is the computation time of one ECDBL + $(n-1)$ ECADDs. The improvement from the method in IEEE is the time of $(n-2)$ ECDBLs $-(2 n+2) / 3$ ECADDs. Moreover, our proposed addition chain computes the scalar multiplication after one ECDBL $+(n-1)$ ECADDs exactly, although the expected time of the binary method is only estimated on average.

The side channel attacks (SCA) allow an adversary to reveal the secret key in the cryptographic device by observing the side channel information such as the computing time and the power consumption [Koc96 KJJ99]. An adversary does not have to break the physical devise to obtain the secret key. It is a serious attack especially against mobile devices like smart cards. The simple power analysis (SPA) only uses a single observed information, while the differential power analysis (DPA) uses a lot of observed information together with statistic tools. There are two approaches to resist the SPA. The first one uses the indistinguishable addition and doubling in the scalar multiplication [CJ01. In the case of prime fields, Hesse and Jacobi form elliptic curves achieve the indistinguishability by using the same formula for both an addition and a doubling [LS01JQ01. Because of the specialty of these curves, they are not compatible to the standardized curves in [ANSIIEEESEC]. The second one uses the add-anddouble-always method to mask the scalar dependency. The Coron's algorithm [Cor99] and the Montgomery form OKS00] are in this category. To resist the DPA, some randomizations are needed Cor99 and an SPA-resistant scheme can be converted to be a DPA-resistant scheme Cor99JT01. The cost of the conversion is relatively cheap comparing with the scalar multiplication itself.

In this paper, we discuss a criteria, which makes our algorithms to be resistant against the SCA by comparing the Coron's algorithm. Moreover, We also propose addition formulas which only use the $x$-coordinates of the points. The computations of the ECADD and the ECDBL require $9 M+3 S$ and $6 M+3 S$, where $M, S$ are the times for a multiplication and a squaring in the definition field $\mathbb{F}_{p}$. By combination of our addition chain and addition formulas, we estab-

\footnotetext{
${ }^{1}$ Recently, Smart proposed a fast implementation over a SIMD type processor, which allows to compute several operations in the definition field in parallel [Sma01].
} 
lish a faster scalar multiplication algorithm resistant against the SCA in both single and parallel computations. The improvement of our scalar multiplication over the previously fastest method is about $37 \%$ for two processors and $5.7 \%$ for a single processor.

\section{Elliptic Curves and Scalar Multiplications}

In this paper we assume that $K=\mathbb{F}_{p}(p>3)$ be a finite field with $p$ elements. Elliptic curves over $K$ can be represented by the equation

$$
E(K):=\left\{(x, y) \in K \times K \mid y^{2}=x^{3}+a x+b\left(a, b \in K, 4 a^{3}+27 b^{2} \neq 0\right)\right\} \cup \mathcal{O},
$$

where $\mathcal{O}$ is the point of infinity. Every elliptic curve is isomorphic to a curve of this form, and we call it the Weierstrass form. An elliptic curve $E(K)$ has an additive group structure. Let $P_{1}=\left(x_{1}, y_{1}\right), P_{2}=\left(x_{2}, y_{2}\right)$ be two elements of $E(K)$ that are different from $\mathcal{O}$ and satisfy $P_{2} \neq \pm P_{1}$. Then the sum $P_{1}+P_{2}=$ $\left(x_{3}, y_{3}\right)$ is defined as follows:

$$
x_{3}=\lambda^{2}-x_{1}-x_{2}, \quad y_{3}=\lambda\left(x_{1}-x_{3}\right)-y_{1},
$$

where $\lambda=\left(y_{2}-y_{1}\right) /\left(x_{2}-x_{1}\right)$ for $P_{1} \neq P_{2}$, and $\lambda=\left(3 x_{1}^{2}+a\right) /\left(2 y_{1}\right)$ for $P_{1}=P_{2}$. We call $P_{1}+P_{2}\left(P_{1} \neq P_{2}\right)$ the elliptic curve addition (ECADD) and $P_{1}+P_{2}\left(P_{1}=P_{2}\right)$, that is $2 * P_{1}$, the elliptic curve doubling (ECDBL). Let $d$ be an integer and $P$ be a point on the elliptic curve $E(K)$. The scalar multiplication is to compute the point $d * P$. There are three types of enhancements of the scalar multiplication. The first one is to represent the elliptic curve $E(K)$ with a different coordinate system, whose scalar multiplication is more efficient. For examples, a projective coordinate and a class of Jacobian coordinate has been studied CMO98. The second one is to use an efficient addition chain. The addition-subtraction chain is an example MO90. We can also apply the addition chains developed for the ElGamal cryptosystem over finite fields Gor98. The third one is to use a special type of curve such as the Montgomery form elliptic curve [OS00], or the Hesse form JQ01 Sma01].

Coordinate System: There are several ways to represent a point on an elliptic curve. The costs of computing an ECADD and an ECDBL depend on the representation of the coordinate system. The detailed description of the coordinate systems is given in [CMO98. The major coordinate systems are as follows: the affine coordinate system $(\mathcal{A})$, the projective coordinate system $(\mathcal{P})$, the Jacobian coordinate system $(\mathcal{J})$, the Chudonovsky coordinate system $\left(\mathcal{J}^{C}\right)$, and the modified Jacobian coordinate system $\left(\mathcal{J}^{m}\right)$. We summarize the costs in Table 1 where $M, S, I$ denotes the computation time of a multiplication, a squaring, and an inverse in the definition field $K$, respectively. The speed of ECADD or ECDBL can be enhanced when the third coordinate is $Z=1$ or the coefficient of the definition equation is $a=-3$. 
Table 1. Computing times of an addition (ECADD) and a doubling (ECDBL)

\begin{tabular}{|c|c|c|c|c|}
\hline \multirow{2}{*}{$\begin{array}{c}\text { Coordinate } \\
\text { System }\end{array}$} & \multicolumn{2}{|c|}{ ECADD } & \multicolumn{2}{c|}{ ECDBL } \\
\cline { 2 - 5 } & $Z \neq 1$ & $Z=1$ & $a \neq-3$ & $a=-3$ \\
\hline $\mathcal{A}$ & $2 M+1 S+1 I$ & - & $2 M+2 S+1 I$ \\
\hline $\mathcal{P}$ & $12 M+2 S$ & $9 M+2 S$ & $7 M+5 S$ & $7 M+3 S$ \\
\hline $\mathcal{J}$ & $12 M+4 S$ & $8 M+3 S$ & $4 M+6 S$ & $4 M+4 S$ \\
\hline $\mathcal{J}^{C}$ & $11 M+3 S$ & $8 M+3 S$ & $5 M+6 S$ & $5 M+4 S$ \\
\hline $\mathcal{J}^{m}$ & $13 M+6 S$ & $9 M+5 S$ & \multicolumn{3}{|c|}{$4 M+4 S$} \\
\hline
\end{tabular}

Addition Chain: Let $d$ be an $n$-bit integer and $P$ be a point of the elliptic curve $E(K)$. A standard way for computing the scalar multiplication $d * P$ is to use the binary expression of $d=d_{n-1} 2^{n-1}+d_{n-2} 2^{n-2}+\ldots+d_{1} 2+d_{0}$, where $d_{n-1}=1$ and $d_{i}=0,1(n=0,1, \ldots, n-2)$. Then Algorithm 1 and Algorithm 2 compute $d * P$ efficiently. We call these methods the binary methods (or the add-and-double methods). On average they require $(n-1)$ ECDBLs $+(n-1) / 2$ ECADDs. Because computing the inverse $-P$ of $P$ is essentially free, we can relax the binary coefficient to a signed binary $d_{i}=-1,0,1(i=0,1, \ldots, n-1)$, which is called the addition-subtraction chain. The NAF offers a way to construct the addition-subtraction chain, which requires $(n-1)$ ECDBLs $+(n-1) / 3$ ECADDs on average [EEE].

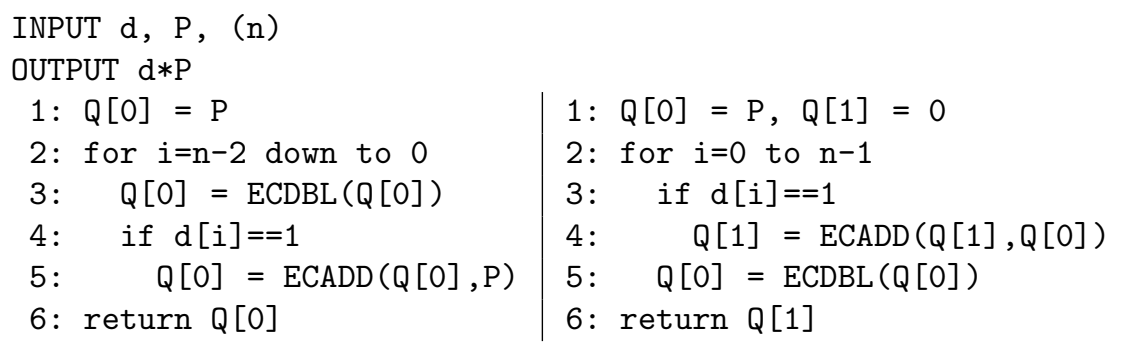

Algorithm 1 (leftside): Binary method from the most significant bit Algorithm 2 (rightside): Binary method from the least significant bit

The other enhancement technique is to utilize pre-computed tables. The Brickell's method and the sliding windows methods are two of the standard algorithms [BSS99]. These algorithms have been developed for the efficient modular multiplications over finite fields. We can refer to the nice survey paper Gor98. In this paper we are interested in efficient algorithms without table look-up (or with a very small pre-computed table). Our goal is to propose an efficient algorithm that is suitable for smart cards, and the pre-computed table sometimes hinders to achieve the high efficiency because the memory spaces are expensive and an I/O interface to read the table is relatively slow.

Special Elliptic Curves: With a special class of elliptic curves, we can enhance the speed of a scalar multiplication. Okeya and Sakurai proposed to use the 
an ECDBL and an ECADD in every bits. Thus an adversary cannot guess the bit information of $d$ by the SPA. A drawback of this method is their efficiency. Algorithm 1' requires $(n-1)$ ECADDs $+(n-1)$ ECDBLs and Algorithm 2' requires $n$ ECADDs $+n$ ECDBLs.

Note that in Algorithm 1' and 2', there are no computational advantage even if we use the NAF because we have to compute both ECADD and ECDBL for each bit.

Möller proposed an SPA-resistant algorithm which is a combination of Algorithm 1' and the window method [Moe01]. However, his method requires extra table look-up (at least three elliptic curve points).

Another countermeasure is to establish the indistinguishability between an ECADD and an ECDBL. Joye, Quisquater and Smart proposed to use the Jacobi and Hesse form elliptic curves, which use the same mathematical formulas for both an ECADD and an ECDBL JQ01 Sma01. As we discussed above, a drawback of this approach is that the Jacobi and Hesse form are special types of elliptic curves and they cannot be used for the standard Weierstrass form.

SPA-Resistance to DPA-Resistance: Even if a scheme is SPA-resistant, it is not always DPA-resistant, because the DPA uses not only a simple power trace but also a statistic analysis, which has been captured by several executions of the SPA. Coron pointed out that some parameters of ECC must be randomized in order to be DPA-resistant [Cor99]. By the randomization we are able to enhance an SPA-resistant scheme to be DPA-resistant.

Coron also proposed three countermeasures, but Okeya and Sakurai showed the bias in his 1st and 2nd countermeasures. They asserted that Coron's 3rd method is secure against the DPA [OS00]. The key idea of Coron's 3rd countermeasure for the projective coordinate is as follows. Note that in the projective coordinate, we require 1 inversion and 2 multiplications in the definition fields to pull back from the projective point $\left(X_{d}: Y_{d}: Z_{d}\right)$ to the affine point $\left(x_{d}, y_{d}\right)$. Let $P=(X: Y: Z)$ be a base point in a projective coordinate. Then $(X: Y: Z)$ equals to $(r X: r Y: r Z)$ for all $r \in K$. If we randomize a base point with $r$ before starting the scalar multiplication, the side information for the statistic analysis will be randomized. This countermeasure requires only three multiplications before the scalar multiplication, and no extra cost after the scalar multiplication.

The other enhancement method against the DPA was proposed by JoyeTymen [JT01. This countermeasure uses an isomorphism of an elliptic curve. The base point $P=(X: Y: Z)$ and the definition parameters $a, b$ of an elliptic curve can be randomized in its isomorphic classes like $\left(r^{2} X: r^{3} Y: Z\right)$ and $r^{4} a, r^{6} b$, respectively. Let $\left(X_{d}^{\prime}: Y_{d}^{\prime}: Z_{d}^{\prime}\right)$ be the point after computing the scalar multiplication. The point $\left(x_{d}, y_{d}\right)$ is pulled back to the original curve by computing $r^{-2} X_{d}^{\prime}$ and $r^{-3} Y_{s}^{\prime}$. This method requires 3 squaring and 5 multiplications for the randomizing the point $P$, and 1 squaring, 3 multiplications, and 1 inversion for pulling back to the original curve. Joye-Tymen method can choose the $Z$-coordinate equal to 1 during the computation of the scalar multiplication and it improves the efficiency of the scalar multiplication in some cases. 


\section{Our Proposed Algorithm}

We explain our proposed algorithm for the scalar multiplication in the following. The algorithm improved on the addition chain and the addition formula. Both improvements are based on the scalar multiplication by Montgomery Mon87. However, we firstly point out that the addition chain is applicable for not only Montgomery form curves but any type of curves. We enhance it to be suitable for implementation and study the security against the SPA compared with Coron's SPA-resistant algorithm (Algorithm 1'). We also establish the addition formulas, which only use the $x$-coordinate of the points, for the Weierstrass form curves.

\subsection{Addition Chain}

We describe our proposed addition chain in the following:

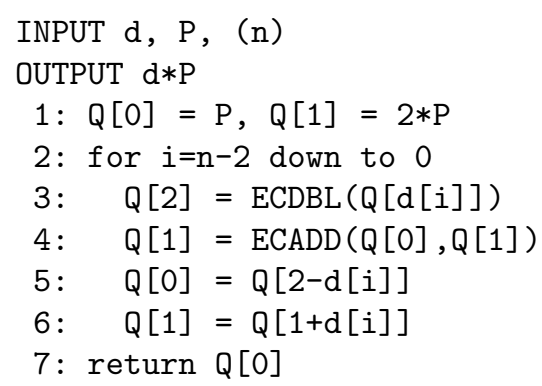

Algorithm 3: Our proposed addition chain (SPA resistant)

For each bit $d[i]$, we compute $Q[2]=\operatorname{ECDBL}(Q[d[i]])$ in Step 3 and $Q[1]=$ $\operatorname{ECADD}(Q[0], Q[1])$ in Step 4. Then the values are assigned $Q[0]=Q[2], Q[1]=$ $Q[1]$ if $d[i]=0$ and $Q[0]=Q[1], Q[1]=Q[2]$ if $d[i]=1$. We prove the correctness of our proposed algorithm in the following.

Theorem 1. Algorithm 3, on input a point $P$ and an integer $d>2$, outputs the correct value of the scalar multiplication $d * P$.

Proof. When we write $Q[0], Q[1]$, it means that $Q[0]$ in Step 5 and $Q[1]$ in Step 6 of Algorithm 3 in the following. The loop of Step 2 generates a sequence

$$
(Q[0], Q[1])_{n-2},(Q[0], Q[1])_{n-3}, \ldots,(Q[0], Q[1])_{1},(Q[0], Q[1])_{0},
$$

from the bit sequence $d[n-2], d[n-3], \ldots, d[1], d[0]$. At first we prove $Q[1]=$ $Q[0]+P$ for each $(Q[0], Q[1])_{i}, i=0,1, . ., n-2$, by the induction for the number of the sequence. For $n=2$ we have only one loop in Step 3 and we have two cases $d[0]=0$ or 1 . Then we obtain $Q[0]=2 * P, Q[1]=3 * P$ for $d[0]=0$, and $Q[0]=3 * P, Q[1]=4 * P$ for $d[0]=1$. The fact $Q[1]=Q[0]+P$ is correct for $n=2$. Next, we assume that $Q[1]=Q[0]+P$ up to $n=k$. In this case we have $R[1]=R[0]+P$, where $(Q[0], Q[1])_{1}=(R[0], R[1])$. For $n=k+1$ we also have two cases $d[0]=0$ or 1 . Then we obtain $Q[0]=2 * R[0], Q[1]=2 * R[0]+P$ for 
$d[0]=0$, and $Q[0]=2 * R[0]+P, Q[1]=2 * R[0]+2 * P$ for $d[0]=1$. The fact $Q[1]=Q[0]+P$ is correct for $n=k+1$. Thus we proved that $Q[1]=Q[0]+P$ for each $(Q[0], Q[1])_{i}, i=0,1, . ., n-2$.

Next, we prove that $Q[0]$ is equivalent to $Q[0]$ in Step 4 of Algorithm $1(Q[0]$ in Step 5 of Algorithm 2) for each loop of $d[i],(i=0,1, \ldots, n-2)$. In each loop of $d[i]$, for given $Q[0], Q[1]$, the new $Q[0]$ is computed as follows: $\operatorname{ECDBL}(Q[0])$ for $d[i]=0$ and $\operatorname{ECADD}(Q[0], Q[1])=Q[0]+(Q[0]+P)=2 * Q[0]+P=$ $\operatorname{ECADD}(\operatorname{ECDBL}(Q[0]), P)$ for $d[i]=1$. On the other hand, in each loop of $d[i]$ in Algorithm 1, for given $Q[0]$, the new $Q[0]$ is computed as follows: $\operatorname{ECDBL}(Q[0])$ for $d[i]=0$ and $\operatorname{ECADD}(\operatorname{ECDBL}(Q[0]), P)$ for $d[i]=1$. They are completely the same computations. Thus we can conclude that the output $d * P$ is correct.

Algorithm 3 requires one ECDBL in the initial Step 1, and $(n-1)$ ECDBLs and $(n-1)$ ECADDs in the loop. The computation time of the loop is same as that of Algorithm 1'.

Remark 1. Algorithm 3 does not depend on the representation of elliptic curves, and it is applicable to execute a modular exponentiation in any abelian group. Therefore the RSA cryptosystem, the DSA, the ElGamal cryptosystem can use our proposed algorithm.

Parallel Computation: First, note that ECADD and ECDBL of each loop of Algorithm 2' can be computed in parallel. Algorithm 2' then requires only $n$ ECADDs with two processors. However, Algorithm 1' cannot be parallelized in this sense, because its loop is constructed from the most significant bit, and the output of ECADD requires the output of ECDBL in each loop. The addition chain of Algorithm 3 is also constructed from the most significant bit, but we can compute the loop of Algorithm 3 in parallel.
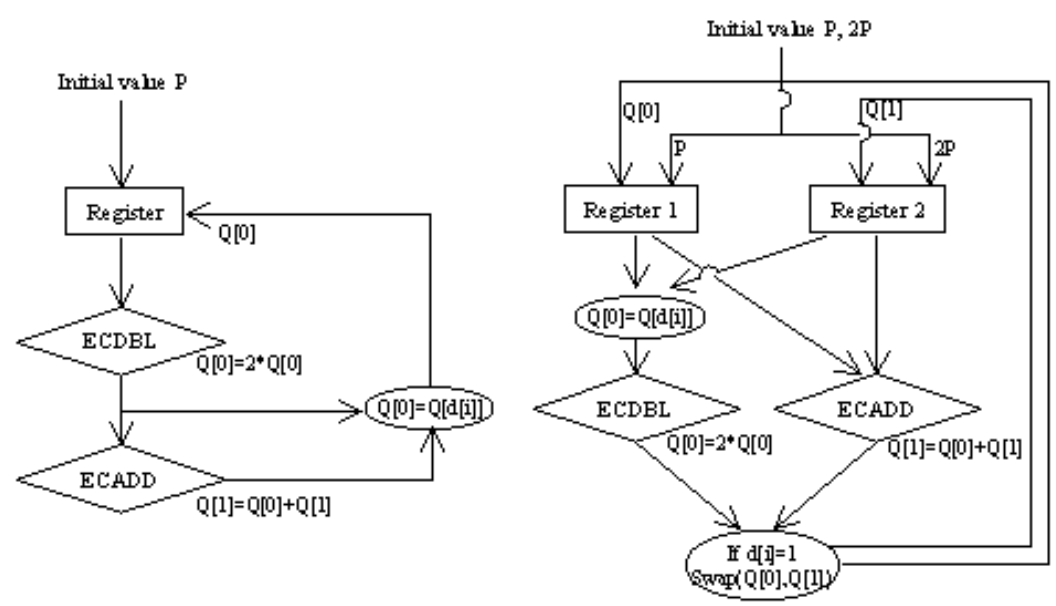

Fig. 1. Algorithm 1' (left), the parallel implementation of Algorithm 3 (right) 
In the right side of Figure 1 we show an architecture of the parallel computation of the loop of Algorithm 3. It has two registers: Register 1 and Register 2, which are initially assigned $Q[0]=P$ and $Q[1]=2 * P$, respectively. In Step 3, we choose the value $Q[d[i]]$ based on the bit information $d[i]$, then compute $\operatorname{ECDBL}(Q[d[i]])$ from $Q[d[i]]$. In Step 4, we compute $\operatorname{ECADD}(Q[0], Q[1])$ from the value $Q[0]$ in Register 1 and $Q[1]$ in Register 2. In both Step 3 and Step 4, they do not need the output from Step 3 nor Step 4, and they are excused independently. After finishing to compute both $\operatorname{ECDBL}(Q[d[i]])$ and $\operatorname{ECADD}(Q[0], Q[1])$, we assign the values in Register 1 and Register 2 based on the bit $d[i]$. If $d[i]=0$, we assign the $\operatorname{ECDBL}(Q[d[i]])$ in Register 1 and the $\operatorname{ECADD}(Q[0], Q[1])$ in Register 2. If $d[i]=1$, we swap the two variables, then we assign the $\operatorname{ECADD}(Q[0], Q[1])$ in Register 1 and the $\operatorname{ECDBL}(Q[d[i]])$ in Register 1.

In general the computation of an ECADD is slower than that of an ECDBL, so that the latency of the loop in Algorithm 3 depends on the running time of ECADDs. Thus the total running time of Algorithm 3 is one ECDBL and $(n-1)$ ECADDs, where $n$ is the bit-length of $d$. Algorithm 1' always requires $(n-1)$ ECDBLs and $(n-1)$ ECADDs. The improvement of Algorithm 3 from Algorithm 1' is $(n-1)$ ECDBLs.

Security Consideration: We discuss the security of Algorithm 3 against the SCA. Algorithm 1' is commonly believed secure against the SPA [OS00]. The relation between Algorithm 1' and Algorithm 3 is as follows.

Theorem 2. Algorithm 3 is as secure as Algorithm 1' against the SPA, if we use a computing architecture whose swapping power of two variables is negligible.

Proof. The differences between Algorithm 1' and Algorithm 3 are Step 5 and Step 6 in Algorithm 3. In the steps, if $d[i]=0$, we assign the $Q[0]=Q[2]$ and $Q[1]=Q[1]$, otherwise, we assign $Q[0]=Q[1]$ and $Q[1]=Q[2]$. We can modify the steps as follows:

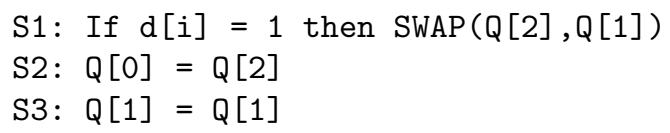

SWAP is a function to swap two variables. Only Step S1 depends on $d[i]$. If the power to execute SWAP is negligible, Algorithm 3 is as secure as Algorithm 1' against the SPA.

Next, an SPA-resistant scheme can be converted to a DPA-resistant scheme using Coron's 3rd or the Joye-Tymen's countermeasure as we discussed in the previous section. Thus, we have the following corollary.

Corollary 1. Algorithm 3 with Coron's 3rd or Joye-Tymen's countermeasure is as secure as Algorithm 1' against the DPA, if we use a computing architecture whose swapping power of two variables is negligible. 
It is possible to implement the swapping of two variables in hardware using a few logic gates. Its power is usually negligible. In software we can implement it just to swap two pointer assignments. The swapping of the pointer assignments in software can be executed in several clocks, whose time or power trace is negligible. Therefore, our proposed method is secure against the DPA in many computing environments.

4-Parallel Computation: When a table of pre-computed points is allowed to be used, we can construct a scalar multiplication, which can be computed in parallel with more than two processors. There are several scalar multiplications using a pre-computed table Gor98. In this paper we are interested in a scalar multiplication with a very small table. The method that uses a very small table is proposed by Lim-Lee [LL94]. The simplest case of the Lim-Lee algorithm requires only one pre-computed value and its improvement over the binary method is $7 / 12$ on average. We review it in the following. Let $d=d_{n-1} 2^{n-1}+d_{n-2} 2^{n-2}+\ldots+$ $d_{1} 2+d_{0}$ be the binary representation of $d$ with $d_{n-1}=1$. Let $k=\lfloor n / 2\rfloor$. The exponent $d$ is represented as

$$
d=2^{k}\left(f[k] 2^{k}+f[k-1] 2^{k-1}+\ldots+f[0] 2^{0}\right)+\left(e[k] 2^{k}+e[k-1] 2^{k-1}+\ldots+e[0] 2^{0}\right),
$$

where $e[i]=d[i], f[i]=d[i+k]$ for $i=0,1, \ldots, k-1, f[k]=e[k]=0$ for even $n$, and $f[k]=1, e[k]=0$ for odd $n$. Then we obtain $d * P=\sum_{i=0}^{k}\left(2^{i}\right) *(e[i] * P+f[i] *$ $\left.\left(\left(2^{k}\right) * P\right)\right)$ and $d * P$ can be computed like in the binary method. This method pre-computes the point $\left(2^{k}\right) * P$ and it is applied for only scalar exponentiations of the fixed based $P$. We modify the Lim-Lee method to be able to compute in parallel and to be secure against the SPA. The proposed algorithm carries 4 auxiliary variables $Q[0][0], Q[0][1], Q[1][0], Q[0][1]$, which are related with

$$
Q[0][1]=Q[0][0]+P, Q[1][0]=Q[0][0]+2^{k} * P, Q[1][1]=Q[0][0]+P+2^{k} * P,
$$

The proposed algorithm is as follows:

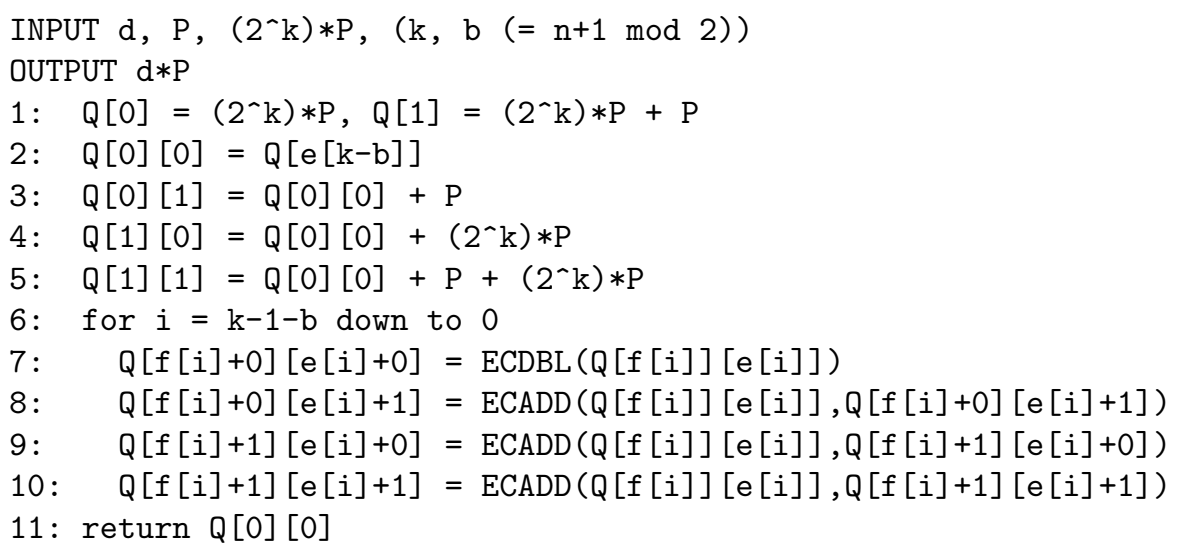

Algorithm 4: Our proposed addition chain II (SPA resistant) 
Due to space limitations, we omit the proof of the correctness of Algorithm 4. When we compute from Step 7 to Step 10 in parallel, the latency of each loop is the time for computing ECADDs. The total number of loops is at most $n / 2-1$, where $n$ is the bit-length of $d$. Therefore Algorithm 4 can be computed at most $(n / 2+3)$ ECADDs with 4 processors. It is about two times faster than Algorithm 3. Moreover, the security against the SPA can be discussed in the same way like Theorem 1 for Algorithm 3. If we use a computing architecture whose swapping powers of four variables are negligible, then Algorithm 4 is secure against the SPA. It is possible to apply Coron's 3rd or Joye-Tymen's countermeasure to make Algorithm 4 secure against the DPA.

\subsection{Addition Formula}

Let $E$ be an elliptic curve defined by the standard Weierstrass form (11) and $P_{1}=\left(x_{1}, y_{1}\right), P_{2}=\left(x_{2}, y_{2}\right), P_{3}=P_{1}+P_{2}=\left(x_{3}, y_{3}\right)$ be points on $E(K)$. Moreover, let $P_{3}^{\prime}=P_{1}-P_{2}=\left(x_{3}^{\prime}, y_{3}^{\prime}\right)$. Then we obtain the following relations:

$$
x_{3} \cdot x_{3}^{\prime}=\frac{\left(x_{1} x_{2}-a\right)^{2}-4 b\left(x_{1}+x_{2}\right)}{\left(x_{1}-x_{2}\right)^{2}}, x_{3}+x_{3}^{\prime}=\frac{2\left(x_{1}+x_{2}\right)\left(x_{1} x_{2}+a\right)+4 b}{\left(x_{1}-x_{2}\right)^{2}} .
$$

On the other hand, letting $P_{4}=2 * P_{1}=\left(x_{4}, y_{4}\right)$ leads to the relation

$$
x_{4}=\frac{\left(x_{1}^{2}-a\right)^{2}-8 b x_{1}}{4\left(x_{1}^{3}+a x_{1}+b\right)} .
$$

Thus the $x$-coordinates of both $P_{3}$ and $P_{4}$ can be computed just form the $x$ coordinates of the points $P_{1}, P_{2}, P_{3}^{\prime}$. We call this method the multiplicative (additive) $x$-coordinate-only method. The $x$-coordinate-only methods for a scalar multiplication were originally introduced by Montgomery [Mon87]. However, his main interest was to find a special form of elliptic curves on which the computing times are optimal. The additive method was not discussed in his paper.

When we use the $x$-coordinate-only methods, we need the difference of two points $P_{3}^{\prime}=P_{1}-P_{2}$. This may be a problem in general, but not in Algorithm 3. In each loop of Algorithm 3, the two points $(Q[0], Q[1])$ are simultaneously computed and they satisfy the equation $Q[1]-Q[0]=P$, where $P$ is a base point of the scalar multiplication. Similarly, in each loop of Algorithm 4, the differences of the points for computing an ECADD are known by equation (15). Therefore, we can assume that the difference $P_{2}-P_{1}$ for input values of $\operatorname{ECADD}\left(P_{1}, P_{2}\right)$ of Algorithm 3 (or Algorithm 4) are always known. On the contrary, in order to know that of Algorithm 2' we need extra computation. The $x$-coordinate-only methods for Algorithm 2' have no computational advantage.

When we apply the $x$-coordinate-only methods to Algorithm 3 (or Algorithm 4), the output is only the $x$-coordinate of $d * P$. This is enough for some cryptographic applications such as a key exchange scheme and an encryption/decryption scheme [SEC]. But other applications also require the $y$ coordinate of $d * P$ in the verification of a signature scheme [SEC]. However, the $y$-coordinate of $d * P$ is easily obtained in the following way: The final values of 
$Q[0], Q[1]$ in Algorithm 3 (or Algorithm 4) are related by $Q[1]=Q[0]+P$. Let $P=\left(x_{1}, y_{1}\right), Q[0]=\left(x_{2}, y_{2}\right), Q[1]=\left(x_{3}, y_{3}\right)$. Here known values are $x_{1}, y_{1}, x_{2}, x_{3}$ and the target is $y_{2}$. Using a standard addition formula (2), we obtain the equation $y_{2}=\left(2 y_{1}\right)^{-1}\left(y_{1}^{2}+x_{2}^{3}+a x_{2}+b-\left(x_{1}-x_{2}\right)^{2}\left(x_{1}+x_{2}+x_{3}\right)\right)$. This $y$-recovering technique was originally introduced by Agnew et al. for curves over $\mathbb{F}_{2^{m}}$ AMV93. The computing time for $y$-recovering is $16 M+4 S+1 I$. 2

In the implementation of the $x$-coordinate-only methods, the projective coordinate system offers a faster computation. In this system, equations (6) and (17) turn to be

$$
\begin{aligned}
\frac{X_{3}}{Z_{3}} & =\frac{Z_{3}^{\prime}}{X_{3}^{\prime}} \frac{\left(X_{1} X_{2}-a Z_{1} Z_{2}\right)^{2}-4 b Z_{1} Z_{2}\left(X_{1} Z_{2}+X_{2} Z_{1}\right)}{\left(X_{1} Z_{2}-X_{2} Z_{1}\right)^{2}} \\
\frac{X_{3}}{Z_{3}} & =\frac{2\left(X_{1} Z_{2}+X_{2} Z_{1}\right)\left(X_{1} X_{2}+a Z_{1} Z_{2}\right)+4 b Z_{1}^{2} Z_{2}^{2}}{\left(X_{1} Z_{2}-X_{2} Z_{1}\right)^{2}}-\frac{X_{3}^{\prime}}{Z_{3}^{\prime}}, \\
\frac{X_{4}}{Z_{4}} & =\frac{\left(X_{1}^{2}-a Z_{1}^{2}\right)^{2}-8 b X_{1} Z_{1}^{3}}{4\left(X_{1} Z_{1}\left(X_{1}^{2}+a Z_{1}^{2}\right)+b Z_{1}^{4}\right)} .
\end{aligned}
$$

The computing times for (18), (99), (10) are $\operatorname{ECADD}_{m}^{(x)}=9 M+2 S, \operatorname{ECADD}_{a}^{(x)}=$ $10 M+2 S, \operatorname{ECDBL}^{(x)}=6 M+3 S$. If $Z_{3}^{\prime}=1$, the computing times deduce to $\operatorname{ECADD}_{m\left(Z_{3}^{\prime}=1\right)}^{(x)}=\operatorname{ECADD}_{a\left(Z_{3}^{\prime}=1\right)}^{(x)}=8 M+2 S$. The concrete algorithms to compute (8), (9), (10) are listed in the appendix.

\section{Comparison}

In this section, we compare the computing times of a scalar multiplication resistant against the SCA. As a result, we show that our proposed algorithm establishes a faster scalar multiplication. The improvement of our scalar multiplication over the previously fastest method is about $37 \%$ for two processors and $5.7 \%$ for a single processor.

Estimation: We compare the computing times of a scalar multiplication with Algorithm 1', 2', and 3 using different coordinate systems. All algorithms are assumed to be DPA-resistant using Coron's 3rd countermeasure or Joye-Tymen's countermeasure, which are described in Section 3 . We estimate the total times to output a scalar multiplication $d * P=\left(x_{d}, y_{d}\right)$ on input $d, P=(x, y)$ and elliptic curve information $(a, b, p)$. The times are given in terms of the numbers of the arithmetic in the definition field, i.e., the multiplication $M$, the squaring $S$, and the inverse $I$. Note that one inversion is always required in order to convert a point from the projective coordinates to the affine coordinates. In the estimation,

\footnotetext{
2 A similar discussion for $y$-recovering on Montgomery form is found in [OS01]. However, Algorithm 2 and Algorithm 4 in [OS01] doesn't output the expected values. The formulas for them must be $X_{d}^{r e c}=4 B y X_{d+1} Z_{d+1} Z_{d} X_{d}, Y_{d}^{\text {rec }}=Z_{d+1}^{2} U^{2}-X_{d+1}^{2} V^{2}$, $Z_{d}^{r e c}=4 B y X_{d+1} Z_{d+1} Z_{d}^{2}$ and $y$-recovering needs only $13 M+1 I$, which is faster than Algorithm 3 in [OS01].
} 
we include the times for randomization by Coron's 3rd countermeasure or JoyeTymen's countermeasure, and the times for recovering the $y$-coordinate in the $x$-coordinate-only method are also included. 3 In the estimation, we also give the estimated running time for a 160-bit scalar. The last numbers in the brackets are the estimation for $1 S=0.8 M, 1 I=30 M$ [OS01].

Single Case: In Table 2, we summarize the estimated running time using a single processor. Algorithm 3/Joye-Tymen with the $x$-coordinate-only methods is the fastest of all scalar multiplications $(2929.0 M)$. The previously fastest algorithm was Algorithm 1'/Joye-Tymen with the Jacobian coordinate system $\mathcal{J}$ $(3095.0 M)$. The improvement of the proposed algorithm over it is about $5.7 \%$.

Table 2. Computing times of a scalar multiplication (a single processor)

\begin{tabular}{|c|c|c|c|}
\hline & \multirow{2}{*}{$\begin{array}{c}\text { Addition } \\
\text { formula }\end{array}$} & \multicolumn{2}{|c|}{ Computing Time } \\
\hline & & Total & $n=160$ \\
\hline \multirow{4}{*}{$\begin{array}{l}\text { Algorithm 1' } \\
\text { /Coron 3rd }\end{array}$} & $\mathcal{P}$ & $(19 n-15) M+(7 n-7) S+1 I$ & $3025 M+1113 S+1 I \quad(3945.4 M)$ \\
\hline & $\mathcal{J}$ & $(16 n-10) M+(10 n-8) S+1 I$ & $2550 M+1592 S+1 I \quad(3853.6 M)$ \\
\hline & $\mathcal{J}^{\mathcal{C}}$ & $(16 n-10) M+(9 n-7) S+1 I$ & $2550 M+1433 S+1 I \quad(3726.4 M)$ \\
\hline & $\mathcal{J}^{m}$ & $(17 n-10) M+(10 n-7) S+1 I$ & $2710 M+1593 S+1 I \quad(4014.4 M)$ \\
\hline \multirow{4}{*}{$\begin{array}{l}\text { Algorithm 1' } \\
\text { /Joye-Tymen }\end{array}$} & $\mathcal{P}$ & $(16 n-7) M+(7 n-4) S+1 I$ & $2553 M+1116 S+1 I \quad(3475.8 M)$ \\
\hline & $\mathcal{J}$ & $(12 n-3) M+(9 n-5) S+1 I$ & $1917 M+1435 S+1 I \quad(3095.0 M)$ \\
\hline & $\mathcal{J}^{\mathcal{C}}$ & $(13 n-4) M+(9 n-5) S+1 I$ & $2076 M+1435 S+1 I \quad(3254.0 M)$ \\
\hline & $\mathcal{J}^{m}$ & $(13 n-4) M+(9 n-5) S+1 I$ & $2076 M+1435 S+1 I \quad(3254.0 M)$ \\
\hline \multirow{4}{*}{$\begin{array}{l}\text { Algorithm 2' } \\
\text { /Coron 3rd }\end{array}$} & $\mathcal{P}$ & $(19 n+4) M+7 n S+1 I$ & $3085 M+1120 S+1 I \quad(4011.0 M)$ \\
\hline & $\mathcal{J}$ & $(16 n+6) M+(10 n+2) S+1 I$ & $2566 M+1602 s+1 I \quad(3877.6 M)$ \\
\hline & $\mathcal{J}^{\mathcal{C}}$ & $(16 n+16) M+(9 n+2) S+1 I$ & $2566 M+1442 S+1 I \quad(3749.6 M)$ \\
\hline & $\mathcal{J}^{m}$ & $(17 n+7) M+(10 n+3) S+1 I$ & $2727 M+1603 S+1 I \quad(4039.4 M)$ \\
\hline \multirow{4}{*}{$\begin{array}{l}\text { Algorithm 2' } \\
\text { /Joye-Tymen }\end{array}$} & $\mathcal{P}$ & $(19 n+9) M+(7 n+3) S+1 I$ & $3049 M+1123 S+1 I \quad(3977.4 M)$ \\
\hline & $\mathcal{J}$ & $(16 n+9) M+(10 n+4) S+1 I$ & $2569 M+1604 S+1 I \quad(3882.2 M)$ \\
\hline & $\mathcal{J}^{\mathcal{C}}$ & $(16 n+9) M+(9 n+4) S+1 I$ & $2569 M+1444 S+1 I \quad(3754.2 M)$ \\
\hline & $\mathcal{J}^{m}$ & $(13 n+9) M+(9 n+4) S+1 I$ & $2089 M+1444 S+1 I \quad(3274.2 M)$ \\
\hline \multirow{6}{*}{$\begin{array}{l}\text { Algorithm } 3 \\
\text { /Coron 3rd }\end{array}$} & $\mathcal{P}$ & $(19 n-8) M+(7 n-2) S+1 I$ & $3032 M+1118 S+1 I \quad(3956.4 M)$ \\
\hline & $\mathcal{J}$ & $(16 n-6) M+(10 n-2) S+1 I$ & $2554 M+1598 S+1 I \quad(3862.4 M)$ \\
\hline & $\mathcal{J}^{\mathcal{C}}$ & $(16 n-5) M+(9 n-1) S+1 I$ & $2555 M+1439 S+1 I \quad(3736.2 M)$ \\
\hline & $\mathcal{J}^{m}$ & $(17 n-6) M+(10 n-3) S+1 I$ & $2714 M+1597 S+1 I \quad(4021.6 M)$ \\
\hline & $x$ (mul) & $(15 n+8) M+(5 n+2) S+1 I$ & $2408 M+802 S+1 I(3079.6 M)$ \\
\hline & $x$ (add) & $(16 n+7) M+(5 n+2) S+1 I$ & $2567 M+802 S+1 I(3238.6 M)$ \\
\hline \multirow{6}{*}{$\begin{array}{l}\text { Algorithm } 3 \\
\text { /Joye-Tymen }\end{array}$} & $\mathcal{P}$ & $(19 n-4) M+7 n S+1 I$ & $3036 M+1120 S+1 I \quad(3962.0 M)$ \\
\hline & $\mathcal{J}$ & $(16 n-4) M+(10 n-1) S+1 I$ & $2556 M+1599 S+1 I \quad(3865.2 M)$ \\
\hline & $\mathcal{J}^{\mathcal{C}}$ & $(16 n-3) M+9 n M+1 I$ & $2557 M+1440 S+1 I \quad(3739.0 M)$ \\
\hline & $\mathcal{J}^{m}$ & $(17 n-5) M+(10 n-3) S+1 I$ & $2715 M+1597 S+1 I \quad(4022.6 M)$ \\
\hline & $x$ (mul) & $(14 n+15) M+(5 n+5) S+1 I$ & $2255 M+805 S+1 I(2929.0 M)$ \\
\hline & $x($ add $)$ & $(14 n+15) M+(5 n+5) S+1 I$ & $2255 M+805 S+1 I(2929.0 M)$ \\
\hline
\end{tabular}

3 These algorithms may contain several inversions, but we can compute them by only one inversion and several multiplications instead. For example, we estimate two inversions $x^{-1}, y^{-1} \in \mathbb{F}_{p}$ as the cost for computing $z=(x y)^{-1}, x^{-1}=z y$ and $y^{-1}=z x$, that is, one inversion and three multiplications. 
In order to demonstrate the efficiency of our algorithm, we implemented our proposed algorithm and the previously fastest algorithm on a Celeron $500 \mathrm{MHz}$ using the LiDIA library [LiDIA]. It should be emphasized here that our implementation was not optimized for cryptographic purposes - it is only intended to provide a comparison. The improvement is about $10 \%$. The results are as follows:

Table 3. Computing times on a Celeron $500 \mathrm{MHz}$ using LiDIA (a single processor)

\begin{tabular}{|l|l|l|}
\hline Previously fastest scheme & Algorithm 1'/Joye-Tymen $(\mathcal{J})$ & $25.5 \mathrm{~ms}$ \\
\hline Proposed scheme & Algorithm 3 /Joye-Tymen $(x)$ & $23.1 \mathrm{~ms}$ \\
\hline
\end{tabular}

Parallel Case: In Table 4, we summarize the estimated running time using two parallel processors. Algorithm 1' cannot be computed in parallel and Algorithm 2' has no computational advantage to use the $x$-coordinate-only methods. Therefore, the previously fastest algorithm was Algorithm 2'/Coron's 3rd with the Chudonovsky coordinate system $\mathcal{J}^{C}(2181.6 M)$. Algorithm 3/Joye-Tymen with $x$-coordinate-only methods provides the fastest multiplication (1593.4M). The improvement of the proposed algorithm over it is about $37 \%$.

Table 4. Computing times of a scalar multiplication (two parallel processors)

\begin{tabular}{|c|c|c|c|}
\hline & \multirow{2}{*}{$\begin{array}{c}\text { Addition } \\
\text { formula }\end{array}$} & \multicolumn{2}{|c|}{ Computing Time } \\
\hline & & Total & $n=160$ \\
\hline \multirow{4}{*}{$\begin{array}{l}\text { Algorithm 2' } \\
\text { /Coron 3rd }\end{array}$} & $\mathcal{P}$ & $(12 n+4) M+2 n S+1 I$ & $1924 M+320 S+1 I \quad(2210.0 M)$ \\
\hline & $\mathcal{J}$ & $(12 n+6) M+(4 n+2) S+1 I$ & $1926 M+642 S+1 I \quad(2469.6 M)$ \\
\hline & $\mathcal{J}^{\mathcal{C}}$ & $(11 n+6) M+(3 n+2) S+1 I$ & $1766 M+482 S+1 I \quad(2181.6 M)$ \\
\hline & $\mathcal{J}^{m}$ & $(13 n+7) M+(6 n+3) S+1 I$ & $2087 M+963 S+1 I \quad(2887.4 M)$ \\
\hline \multirow{4}{*}{$\begin{array}{l}\text { Algorithm 2' } \\
\text { /Joye-Tymen }\end{array}$} & $\mathcal{P}$ & $(12 n+9) M+(2 n+3) S+1 I$ & $1929 M+323 S+1 I \quad(2217.4 M)$ \\
\hline & $\mathcal{J}$ & $(12 n+9) M+(4 n+4) S+1 I$ & $1929 M+644 S+1 I$ \\
\hline & $\mathcal{J}^{\mathcal{C}}$ & $(11 n+9) M+(3 n+4) S+1 I$ & $1769 M+484 S+1 I \quad(2186.2 M)$ \\
\hline & $\mathcal{J}^{m}$ & $(13 n+9) M+(6 n+4) S+1 I$ & $2089 M+964 S+1 I \quad(2890.2 M)$ \\
\hline \multirow{6}{*}{$\begin{array}{l}\text { Algorithm } 3 \\
\text { /Coron 3rd }\end{array}$} & $\mathcal{P}$ & $(12 n-1) M+(2 n+3) S+1 I$ & $1919 M+323 S+1 I \quad(2207.4 M)$ \\
\hline & $\mathcal{J}$ & $(12 n-2) M+(4 n+4) S+1 I$ & $1918 M+644 S+1 I \quad(2463.2 M)$ \\
\hline & $\mathcal{J}^{\mathcal{C}}$ & $11 n M+(3 n+5) S+1 I$ & $1760 M+485 S+1 I \quad(2178.0 M)$ \\
\hline & $\mathcal{J}^{m}$ & $(13 n-2) M+(6 n+1) S+1 I$ & $2078 M+961 S+1 I$ \\
\hline & \begin{tabular}{|l}
$x$ (mul) \\
\end{tabular} & $(9 n+14) M+(2 n+5) S+1 I$ & $1454 M+325 S+1 I(1744.0 M)$ \\
\hline & $x$ (add) & $(10 n+13) M+(2 n+5) S+1 I$ & $1613 M+325 S+1 I(1903.0 M)$ \\
\hline \multirow{6}{*}{$\begin{array}{l}\text { Algorithm } 3 \\
\text { /Joye-Tymen }\end{array}$} & $\mathcal{P}$ & $(12 n+3) M+(2 n+5) S+1 I$ & $1923 M+325 S+1 I \quad(2213.0 M)$ \\
\hline & $\mathcal{J}$ & $12 n M+(4 n+5) S+1 I$ & $1920 M+645 S+1 I$ \\
\hline & $\mathcal{J}^{\mathcal{C}}$ & $(11 n+2) M+(3 n+6) S+1 I$ & $1762 M+486 S+1 I$ \\
\hline & $\mathcal{J}^{m}$ & $(13 n-1) M+(6 n+1) S+1 I$ & $2079 M+961 S+1 I \quad(2877.8 M)$ \\
\hline & $\boldsymbol{x}$ (mul) & $(8 n+21) M+(2 n+8) S+1 I$ & $1301 M+328 S+1 I(1593.4 M)$ \\
\hline & $x$ (add) & $(8 n+21) M+(2 n+8) S+1 I$ & $1301 M+328 S+1 I(1593.4 M)$ \\
\hline
\end{tabular}




\section{Acknowledgments}

We would like to thank Edlyn Teske, Bodo Möller, and Evangelos Karatsiolis for their valuable comments, and the anonymous referees for their helpful comments.

\section{References}

AMV93. G.Agnew, R.Mullin and S.Vanstone, "An implementation of elliptic curve cryptosystems over $F_{2155}$ ", IEEE Journal on Selected Areas in Communications, vol.11, pp.804-813, 1993.

ANSI. ANSI X9.62, Public Key Cryptography for the Financial Services Industry: The Elliptic Curve Digital Signature Algorithm (ECDSA), draft, 1998.

BSS99. I.Blake, G.Seroussi and N.Smart, Elliptic Curves in Cryptography, Cambridge University Press, 1999.

Cor99. J.Coron, "Resistance against differential power analysis for elliptic curve cryptosystems", CHES'99, LNCS 1717, pp.292-302, Springer-Verlag, 1999.

CMO98. H.Cohen, A.Miyaji and T.Ono, "Efficient elliptic curve exponentiation using mixed coordinates", Asiacrypt'98, LNCS 1514, pp.51-65, Springer-Verlag, 1998.

CJ01. C.Clavier and M.Joye, "Universal exponentiation algorithm - A first step towards provable SPA-resistance -", CHES2001, LNCS 2162, pp.300-308, SpringerVerlag, 2001.

Gor98. D.Gordon, "A survey of fast exponentiation methods", J. Algorithms, vol.27, pp.129-146, 1998.

IEEE. IEEE P1363, Standard Specifications for Public-Key Cryptography, 2000. Available from http://groupe.ieee.org/groups/1363/

JQ01. M. Joye and J. Quisquater, "Hessian elliptic curves and side-channel attacks", CHES2001, LNCS 2162, pp.402-410, Springer-Verlag, 2001.

JT01. M.Joye and C.Tymen, "Protections against differential analysis for elliptic curve cryptography", CHES2001, LNCS 2162, pp.377-390, Springer-Verlag, 2001.

Koc96. C.Kocher, "Timing attacks on Implementations of Diffie-Hellman, RSA, DSS, and other systems", Crypto'96, LNCS 1109, pp.104-113, Springer-Verlag, 1996.

KJJ99. C.Kocher, J.Jaffe and B.Jun, "Differential power analysis", Crypto'99, LNCS 1666, pp.388-397, Springer-Verlag, 1999.

LiDIA. LiDIA, A C++ Library For Computational Number Theory, Technische Universtät Darmstadt, http://www.informatik.tu-darmstadt.de/TI/LiDIA/

LS01. P.Liardet and N.Smart, "Preventing SPA/DPA in ECC systems using the Jacobi form", CHES2001, LNCS 2162, pp.391-401, Springer-Verlag, 2001.

LL94. C. Lim and P. Lee, "More flexible exponentiation with precomputation", Crypto'94, LNCS 839, p.95-107, Springer-Verlag, 1994.

Moe01. B.Möller, "Securing elliptic curve point multiplication against side-channel attacks", ISC 2001, LNCS 2200. p.324-334, Springer-Verlag, 2001.

Mon87. P.Montgomery, "Speeding the Pollard and elliptic curve methods for factorizations", Math. of Comp, vol.48, pp.243-264, 1987.

MO90. F. Morain and J. Olivos, "Speeding up the computation on an elliptic curve using addition-subtraction chains", Inform. Theory Appl. 24, pp.531-543, 2000.

NIST. National Institute of Standards and Technology, Recommended Elliptic Curves for Federal Government Use, in the appendix of FIPS 186-2, Available from http://csrc.nist.gov/publication/fips/fips186-2/fips186-2.pdf 
OKS00. K.Okeya, H.Kurumatani and K.Sakurai, "Elliptic curves with the Montgomery form and their cryptographic applications", PKC2000, LNCS 1751, pp.446465, Springer-Verlag, 2000.

OS00. K.Okeya and K.Sakurai, "Power analysis breaks elliptic curve cryptosystems even secure against the timing attack", Indocrypt 2000, LNCS 1977, pp.178-190, Springer-Verlag, 2000.

OS01. K.Okeya and K.Sakurai, "Efficient elliptic curve cryptosystems from a scalar multiplication algorithm with recovery of the $y$-coordinate on a Montgomery-form elliptic curve", CHES2001, LNCS 2162, pp.126-141, Springer-Verlag, 2001.

Sma01. N.Smart, "The Hessian form of an elliptic curve", CHES2001, LNCS 2162, pp.118-125, Springer-Verlag, 2001.

SEC. Standards for Efficient Cryptography Group (SECG), Specification of Standards for Efficient Cryptography. Available from http://www.secg.org

WAP. Wireless Application Protocol (WAP) Forum, Wireless Transport Layer Security (WTLS) Specification. Available from http://www. wapforum.org

\section{Appendix}

The appendix describes the formulas of $\operatorname{ECDBL}^{(x)}, \operatorname{ECADD}_{m}^{(x)}$, and $\operatorname{ECADD}_{a}^{(x)}$, which are proposed in Section 5. In order to estimate the efficiency, we use three notations $\times, \cdot, *$ for the multiplication of the definition field $K$. The notation $\times$ is a standard multiplication in $K$. The notation - is executed in negligible time. The notation $*$ is also calculated in negligible time if we choose $Z_{3}^{\prime}=1$.

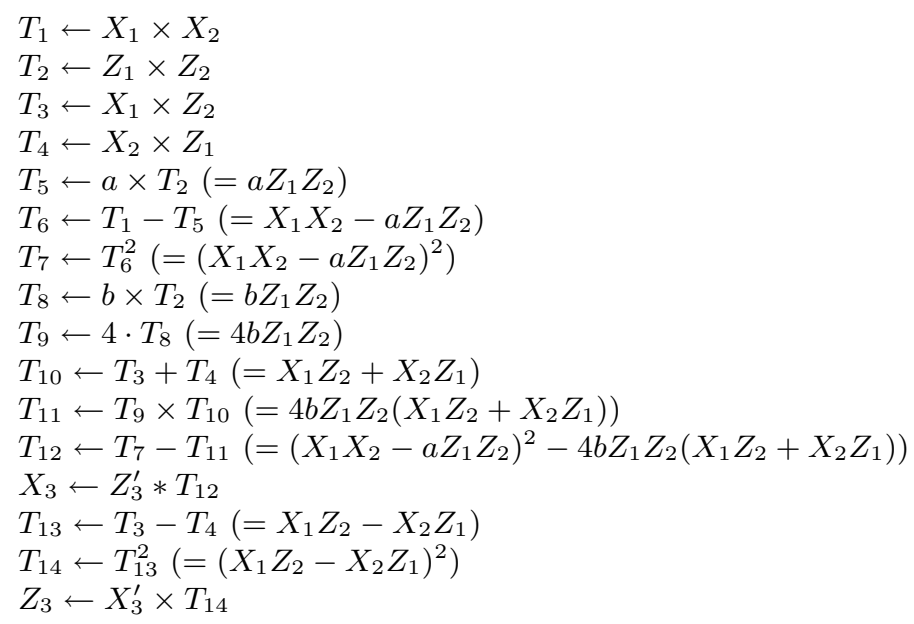

Formula 1. Computing $\operatorname{ECADD}_{m}^{(x)}\left(\cdot\right.$ is negligible, $*$ is negligible if $\left.Z_{3}^{\prime}=1\right)$ 


$$
\begin{aligned}
& T_{1} \leftarrow X_{1} \times X_{2} \\
& T_{2} \leftarrow Z_{1} \times Z_{2} \\
& T_{3} \leftarrow X_{1} \times Z_{2} \\
& T_{4} \leftarrow X_{2} \times Z_{1} \\
& T_{5} \leftarrow T_{3}+T_{4}\left(=X_{1} Z_{2}+X_{2} Z_{1}\right) \\
& T_{6} \leftarrow a \times T_{2}\left(=a Z_{1} Z_{2}\right) \\
& T_{7} \leftarrow T_{1}+T_{6}\left(=X_{1} X_{2}+a Z_{1} Z_{2}\right) \\
& T_{8} \leftarrow T_{5} \times T_{7}\left(=\left(X_{1} Z_{2}+X_{2} Z_{1}\right)\left(X_{1} X_{2}+a Z_{1} Z_{2}\right)\right) \\
& T_{9} \leftarrow 2 \cdot T_{8}\left(=2\left(X_{1} Z_{2}+X_{2} Z_{1}\right)\left(X_{1} X_{2}+a Z_{1} Z_{2}\right)\right) \\
& T_{10} \leftarrow T_{2}^{2}\left(=Z_{1}^{2} Z_{2}^{2}\right) \\
& T_{11} \leftarrow b \times T_{10}\left(b Z_{1}^{2} Z_{2}^{2}\right) \\
& T_{12} \leftarrow 4 \cdot T_{11}\left(=4 b Z_{1}^{2} Z_{2}^{2}\right) \\
& T_{13} \leftarrow T_{9}+T_{12}\left(=2\left(X_{1} Z_{2}+X_{2} Z_{1}\right)\left(X_{1} X_{2}+a Z_{1} Z_{2}\right)+4 b Z_{1}^{2} Z_{2}^{2}\right) \\
& T_{14} \leftarrow T_{3}-T_{4}\left(=X_{1} Z_{2}-X_{2} Z_{1}\right) \\
& T_{15} \leftarrow T_{14}^{2}\left(=\left(X_{1} Z_{2}-X_{2} Z_{1}\right)^{2}\right) \\
& T_{16} \leftarrow Z_{3}^{\prime} * T_{13} \\
& T_{17} \leftarrow X_{3}^{\prime} \times T_{15} \\
& X_{3} \leftarrow T_{16}-T_{17} \\
& Z_{3} \leftarrow Z_{3}^{\prime} * T_{15}
\end{aligned}
$$

Formula 2. Computing $\operatorname{ECADD}_{a}^{(x)}\left(\cdot\right.$ is negligible, $*$ is negligible if $\left.Z_{3}^{\prime}=1\right)$

$$
\begin{aligned}
& T_{1} \leftarrow X_{1}^{2} \\
& T_{2} \leftarrow Z_{1}^{2} \\
& T_{3} \leftarrow a \times T_{2}\left(=a Z_{1}^{2}\right) \\
& T_{4} \leftarrow T_{1}-T_{3}\left(=X_{1}^{2}-a Z_{1}^{2}\right) \\
& T_{5} \leftarrow T_{4}^{2}\left(=\left(X_{1}^{2}-a Z_{1}^{2}\right)^{2}\right) \\
& T_{6} \leftarrow b \times T_{2}\left(=b Z_{1}^{2}\right) \\
& T_{7} \leftarrow X_{1} \times Z_{1}\left(=X_{1} Z_{1}\right) \\
& T_{8} \leftarrow T_{6} \times T_{7}\left(=b X_{1} Z_{1}^{3}\right) \\
& T_{9} \leftarrow 8 \cdot T_{8}\left(=8 b X_{1} Z_{1}^{3}\right) \\
& X_{4} \leftarrow T_{5}-T_{9} \\
& T_{10} \leftarrow T_{1}+T_{3}\left(=X_{1}^{2}+a Z_{1}^{2}\right) \\
& T_{11} \leftarrow T_{7} \times T_{10}\left(=X_{1} Z_{1}\left(X_{1}^{2}+a Z_{1}^{2}\right)\right) \\
& T_{12} \leftarrow T_{6} \times T_{2}\left(=b Z_{1}^{4}\right) \\
& \left.T_{13} \leftarrow T_{11}+T_{12}\left(=X_{1} Z_{1}\left(X_{1}^{2}+a Z_{1}^{2}\right)+b Z_{1}^{4}\right)\right) \\
& Z_{4} \leftarrow 4 \cdot T_{13}
\end{aligned}
$$

Formula 3. Computing $\operatorname{ECDBL}^{(x)}$ ( $\cdot$ is negligible) 